\title{
Variability of the Spectral Energy Distribution of the Blazar S5 0716+714
}

\author{
B. Rani ${ }^{1 *}$, Alok C. Gupta ${ }^{1}$, Paul J. Wiita ${ }^{2}$ \\ ${ }^{1}$ Aryabhatta Research Institute of Observational Sciences (ARIES), Nainital, 263129, India \\ ${ }^{2}$ Department of Physics, The College of New Jersey, P.O. Box 7718, Ewing, NJ 08628, USA
}

Accepted . Received ; in original form 2010 November 24

\begin{abstract}
The emission from blazars is known to be variable at all wavelengths. The flux variability is often accompanied by spectral changes. Spectral energy distribution (SED) changes must be associated with changes in the spectra of emitting electrons and/or the physical parameters of the jet. Meaningful modeling of blazar broadband spectra is required to understand the extreme conditions within the emission region. Not only is the broadband SED crucial, but also information about its variability is needed to understand how the highest states of emission occur and how they differ from the low states. This may help in discriminating between models. Here we present the results of our SED modeling of the blazar S5 $0716+714$ during various phases of its activity. The SEDs are classified into different bins depending on the optical brightness state of the source.
\end{abstract}

\section{Keywords}

galaxies: active - galaxies: quasars: individual: S5 $0716+714$

\section{Introduction}

S5 0716+714 is a bright, high declination BL Lac object at a redshift, $z=0.31 \pm 0.08$ (Nilsson et al. 2008). This source has been extensively studied across the whole electromagnetic spectrum and exhibits strong variability on a wide range of timescales, ranging from minutes to years (e.g., Wagner et al. 1990; Heidt \& Wagner 1996; Villata et al. 2000; Raiteri et al. 2003; Montagni et al. 2006; Ostorero et al. 2006; Gupta et al. 2008a, b, 2009 and references therein). Nearly periodic oscillations of $\sim 15$ minutes in the optical R band were detected in this source (Rani et al. 2010). The optical duty cycle of S5 $0716+714$ is nearly unity, indicating that the source is always in an active state in the visible (Wagner \& Witzel 1995). This blazar was recently shown to be a strong source in the high energy gamma-ray band by FermiLAT (Abdo et al. 2009).

\section{Multi-frequency Data}

We carried out this study of the SEDs of the BL Lac object S5 $0716+714$ using high quality multifrequency data in the literature. The data cover radio to optical energy bands. The optical U, B, V, R and I fluxes were collected from Raiteri et al. (2003), Montagni et al. (2006), Villata et al. (2000), Ostorero et al. (2006) and Gu et al. (2006). The data at radio frequencies of 22 and $37 \mathrm{GHz}$ were taken from Salonen et al. (1987), Teräsranta et al. (1992, 1998, 2004, 2005). The $\mathrm{UMRAO}^{1}$ (University of Michigan Radio Astronomy Observatory) data at 4.8, 8 and $14.5 \mathrm{GHz}$ frequencies was provided by Margo Aller. The data at optical frequencies spans over a period of 1994 November through 2004 April while the radio data cover a time period of 1991 September to 2005 May (Fig. 1).

We model the SEDs of S5 0716+714 using the above radio to optical frequency data. The long term data allow us to obtain six different mean SEDs of the source corresponding to different phases of its activity (Fig. 2). These six different SEDs are characterized by different optical outburst phases and the radio data is averaged over the same time periods.

\footnotetext{
*E-mail: bindu@aries.res.in
}

\footnotetext{
${ }^{1}$ http://www.astro.lsa.umich.edu/obs/radiotel/umrao.php
} 


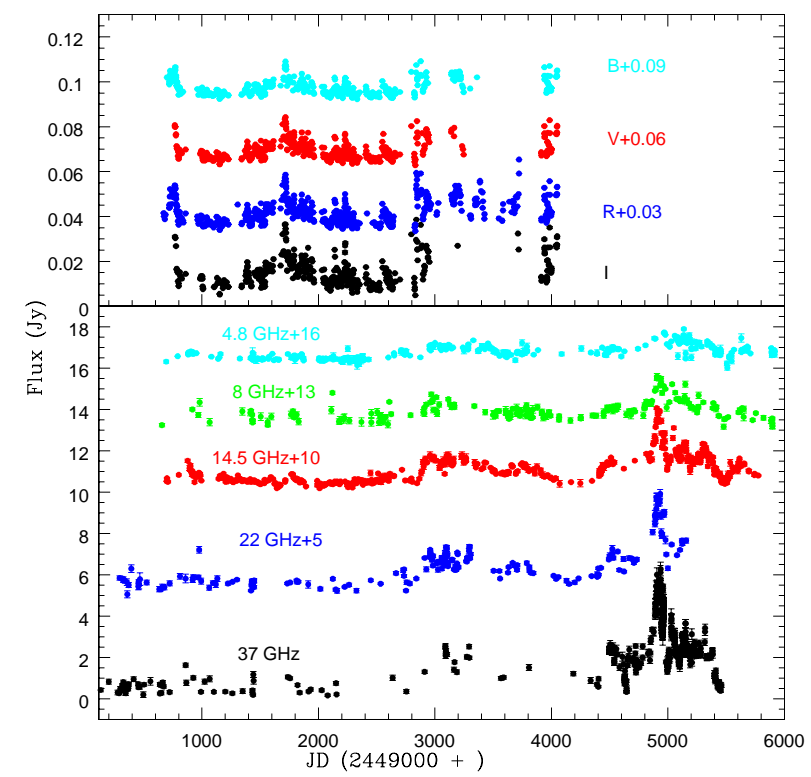

Figure 1. The multi-frequency radio and optical data of the BL Lac object S5 0716+714.

\section{Analysis and Results}

\subsection{SED Modeling}

We use a homogeneous synchrotron self-compton model (SSC) with a broken power law (BPL) to fit the lower energy part of observed spectra which are characterized by synchrotron spectra. The SED model fitting is achieved by using a SED code available online $^{2}$. The best fit model was obtained by varying the parameters of a numerical SSC code (Tramacere 2007; Tramacere et al. 2009). The model assumes that radiation is produced within a single zone of jet ( $\sim$ radius $R)$, moving relativistically at a small angle to the line of sight of the observer. The observed radiation is amplified via the boosting factor $\delta=[\Gamma(1-\beta \cos \theta)]^{-1}$.

Below and above the peaks, the spectrum can be approximated with power law profiles with indices $\alpha_{1}$ and $\alpha_{2}$, respectively. The power law spectra in AGNs are naturally produced if the emitting electron follow a power-law distribution in energy. We approximate this behaviour with a broken power law

\footnotetext{
${ }^{2}$ http://tools.asdc.asi.it/
}

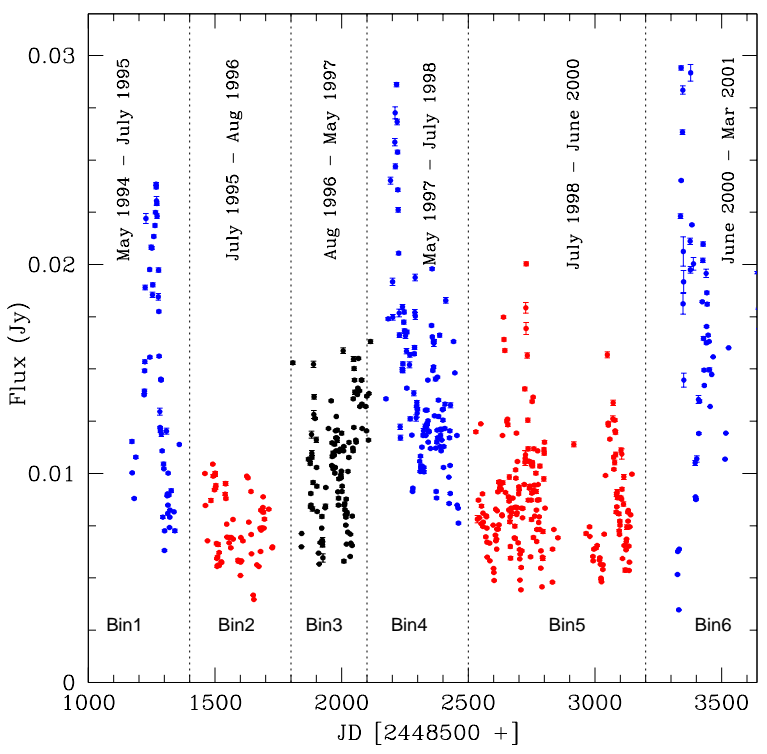

Figure 2. The optical R passband flux of S5 0716+714 divided into six different bins on the basis of its optical activity. Bin1, Bin4 and Bin6 represent the optical outburst phases while Bin2, Bin3 and Bin5 represent dimmer phases of the source.

(BPL) with indices $n_{1}$ and $n_{2}$, respectively below and above the break energy $\Gamma_{b} \mathrm{~m}_{e} \mathrm{c}^{2}$ :

$$
P(\gamma)= \begin{cases}N \Gamma^{-n_{1}} & \text { if } \Gamma<\Gamma_{b} \\ N \Gamma_{b}^{n_{2}-n_{1}} \Gamma^{-n_{2}} & \text { if } \Gamma>\Gamma_{b}\end{cases}
$$

With these approximations, we can completely specify the model with the following parameters: magnetic field intensity (B), size of emission region (R), Doppler boosting factor $(\delta)$, power-law indices $\left(\mathrm{n}_{1}\right.$, $\mathrm{n}_{2}$ ), number density of emitting electrons $(\mathrm{N})$ and Lorentz factor of the electrons at the break energy $\left(\Gamma_{b}\right)$. The values of all these fitted parameters for different SEDs are listed in Table 1.

\section{Discussion and Conclusions}

\subsection{Limitations to the model}

Although, as seen in Fig. 3, we were able to achieve reasonably good fits of the synchrotron emission for all six SEDs of the source, one should bear in mind that the one-zone BPL model is over-simplified in 


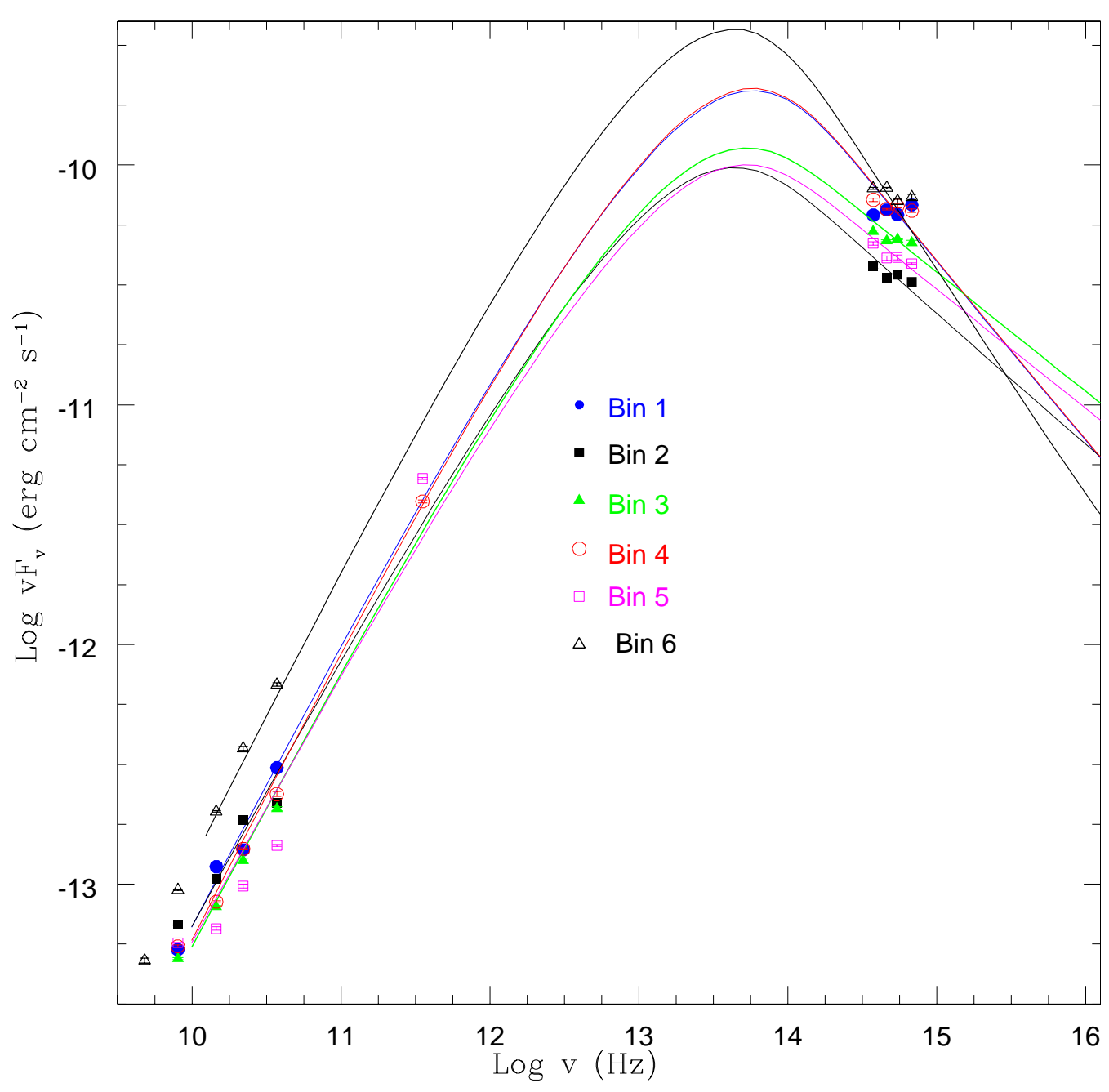

Figure 3. The SSC modeled SEDs of the source corresponding to six different Bins.

accounting for the radio-optical blazar emission. The applicability of a single-zone emitting region has been questioned by a number of authors (e.g., Vittorini et al. 2009, Raiteri et al. 2010). They showed that the BL Lac SED can be more successfully modeled with two synchrotron components (two different emitting populations).

Furthermore, unfortunately, we do not have synchrotron peak measurements for the the source, which would significantly help to constrain the model. Another limitation to the model is that we do not correct our optical measurements for any possible host galaxy contribution. Some objects, such as BL Lac itself, have a significant contribution from starlight to the optical bands, which will modify the calculated synchrotron emission in this region, especially during the low states. We also do not make any corrections for Galaxy or internal absorption, which may again slightly affect the optical-UV part of the spectra. Last, but not least, we stress that our data is not strictly simultaneous but has been averaged over a period of months. As blazars are highly variable over timescales of a day or less, the time differences 
Table 1. Fitted parameters of SED model

\begin{tabular}{|c|c|c|c|c|c|c|c|c|c|c|c|}
\hline & $\begin{array}{l}\log R \\
(\mathrm{~cm})\end{array}$ & $\begin{array}{c}\text { B } \\
\text { (Gauss) }\end{array}$ & $\delta$ & $\begin{array}{c}\mathrm{N} \\
\left(\mathrm{cm}^{-3}\right)\end{array}$ & $\log \Gamma_{\min }$ & $\log \Gamma_{\max }$ & $n_{1}$ & $n_{2}$ & $\log \Gamma_{b}$ & $\begin{array}{c}\log \nu_{\text {peak }} \\
(\mathrm{Hz})\end{array}$ & $\begin{array}{c}\log \nu F_{\nu \text { peak }} \\
\left(\operatorname{erg~cm}^{-2} \mathrm{~s}^{-1}\right)\end{array}$ \\
\hline Bin1 & 16.8 & 0.4 & 12.8 & 25 & 0.2 & 6 & 0.4 & 4.5 & 3.20 & 13.76 & -9.69 \\
\hline $\operatorname{Bin} 2$ & 16.8 & 0.4 & 11.5 & 30 & 0.2 & 6 & 0.6 & 4.1 & 3.14 & 13.61 & -10.01 \\
\hline $\operatorname{Bin} 3$ & 16.8 & 0.4 & 11.0 & 30 & 0.2 & 6 & 0.5 & 4.0 & 3.18 & 13.75 & -9.92 \\
\hline Bin 4 & 16.8 & 0.4 & 12.5 & 25 & 0.2 & 6 & 0.3 & 4.5 & 3.20 & 13.76 & -9.67 \\
\hline Bin5 & 16.8 & 0.4 & 11.0 & 30 & 0.2 & 6 & 0.6 & 4.0 & 3.18 & 13.72 & -9.99 \\
\hline Bin6 & 16.8 & 0.4 & 16.0 & 25 & 0.2 & 6 & 0.2 & 4.9 & 3.20 & 13.64 & -9.42 \\
\hline
\end{tabular}

$\mathrm{R}$ : Size of emitting region

B : Magnetic field

$\delta$ : Doppler boosting factor

$\mathrm{N}$ : Number density of emitting electrons

$\Gamma_{\min }, \Gamma_{\max }:$ Minimum and maximum values of Lorentz factor

$\Gamma_{b}$ : Lorentz factor corresponding to break energy of electrons

$n_{1}, n_{2}$ : Power law indices

$\nu_{\text {Peak }}:$ Synchrotron peak frequency

and averaging might have compromised somewhat the modeling.

\subsection{Spectral Energy Distribution variation}

To attempt a study of how the physical parameters related to emission region and synchrotron emission are changed when the BL Lac S5 0716+714 goes through various phases of its activity we used the simplest approach by fitting a single-zone SSC model. The observed results can be summarized as :

1 . No change between the R and B bands in the modeled SED are seen during different phases of activity. 2. The Doppler boosting factor $\delta$ is higher during the optically bright states of the source compared to the dimmer phases of activity.

3. The number density $(\mathrm{N})$ of electrons emitting synchrotron photons is larger when the source is in lower states.

4. The synchrotron peak frequency $\left(\nu_{\text {peak }}\right)$ and peak intensity $\left(\nu F_{\nu \text { peak }}\right)$ are comparatively higher during the optical outburst phases of the BL Lac object.

This research was supported by CSIR Foreign travel grant Ref No. TG/5295/1-HRD and has made use of data from the University of Michigan Radio Astronomy Observatory which has been supported by the University of Michigan and by a series of grants from the National Science Foundation, most recently AST-0607523. BR is very thankful to Margo Aller for providing the radio frequency data.

\section{References}

Abdo, A. A., et al. 2009, ApJ, 707, 1310

Gupta, A. C., et al. 2008a, AJ, 136, 2359

Gupta, A. C., Fan, J. H., Bai, J. M., \& Wagner, S. J. 2008b, AJ, 135, 1384

Gupta, A. C., Srivastava, A. K., \& Wiita, P. J. 2009, ApJ, 690, 216

Gu, M. F., Lee, C.-U., Pak, S., Yim, H. S., \& Fletcher, A. B. 2006, A\&A, 450, 39

Heidt, J., \& Wagner, S. J. 1996, A\&A, 305, 42

Montagni, F., Maselli, A., Massaro, E., Nesci, R., Sclavi, S., \& Maesano, M. 2006, A\&A, 451, 435

Nilsson, K., Pursimo, T., Sillanpää, A., Takalo, L. O., \& Lindfors, E. 2008, A\&A, 487, L29

Ostorero, L., et al. 2006, A\&A, 451, 797

Raiteri, C. M., et al. 2003, A\&A, 402, 151

Raiteri, C. M., et al. 2010, arXiv:1009.2604

Rani, B., Gupta, A. C., Joshi, U. C., Ganesh, S., \& Wiita, P. J. 2010, ApJ, 719, L153

Salonen, E., et al. 1987, A\&AS, 70, 409

Tramacere, A., Giommi, P., Perri, M., Verrecchia, F., \& Tosti, G. 2009, A\&A, 501, 879

Tramacere, A., Massaro, F., \& Cavaliere, A. 2007, A\&A, 466, 521

Teräsranta, H., et al. 1992, A\&AS, 94, 121

Teräesranta, H., et al. 1998, A\&AS, 132, 305

Teräsranta, H., et al. 2004, A\&A, 427, 769

Teräsranta, H., Wiren, S., Koivisto, P., Saarinen, V., \& Hovatta, T. 2005, A\&A, 440, 409

Villata, M., et al. 2000, A\&A, 363, 108

Vittorini, V., et al. 2009, ApJ, 706, 1433

Wagner, S., Sanchez-Pons, F., Quirrenbach, A., \& Witzel, A. 1990, A\&A, 235, L1

Wagner, S. J., \& Witzel, A. 1995, ARA\&A, 33, 163 